\title{
Analysis of the Elastic Large Deflection Behavior for Metal Plates under Nonuniformly Distributed Lateral Pressure with In-Plane Loads
}

\author{
Jeom Kee Paik, Ju Hye Park, and Bong Ju Kim \\ The Ship and Offshore Research Institute, The Lloyd's Register Educational Trust Research Centre of \\ Excellence, Pusan National University, Busan 609-735, Republic of Korea \\ Correspondence should be addressed to Jeom Kee Paik, jeompaik@pusan.ac.kr
}

Received 21 February 2012; Accepted 22 August 2012

Academic Editor: Carl M. Larsen

Copyright (C) 2012 Jeom Kee Paik et al. This is an open access article distributed under the Creative Commons Attribution License, which permits unrestricted use, distribution, and reproduction in any medium, provided the original work is properly cited.

\begin{abstract}
The Galerkin method is applied to analyze the elastic large deflection behavior of metal plates subject to a combination of in-plane loads such as biaxial loads, edge shear and biaxial inplane bending moments, and uniformly or nonuniformly distributed lateral pressure loads. The motive of the present study was initiated by the fact that metal plates of ships and ship-shaped offshore structures at sea are often subjected to non-uniformly distributed lateral pressure loads arising from cargo or water pressure, together with inplane axial loads or inplane bending moments, but the current practice of the maritime industry usually applies some simplified design methods assuming that the non-uniform pressure distribution in the plates can be replaced by an equivalence of uniform pressure distribution. Applied examples are presented, demonstrating that the current plate design methods of the maritime industry may be inappropriate when the non-uniformity of lateral pressure loads becomes more significant.
\end{abstract}

\section{Introduction}

Ships and ship-shaped offshore structures are composed of metal plate elements, and the accurate computation of nonlinear behavior of the plate elements in deck, bottom and side shells up to the ultimate limit state is a basic requirement for the structural safety assessment. The plate elements in ships and ship-shaped offshore structures are generally subjected to combined inplane and lateral pressure loads. Inplane loads include biaxial compression/tension, biaxial inplane bending and edge shear, as shown in Figure 1, which are mainly induced by overall hull girder bending and/or torsion of the vessel. Lateral pressure loads are due to water pressure and/or cargo. In rough weather, roll and/or pitch motions of vessels are typical as shown in Figure 2, and, subsequently, the distribution 


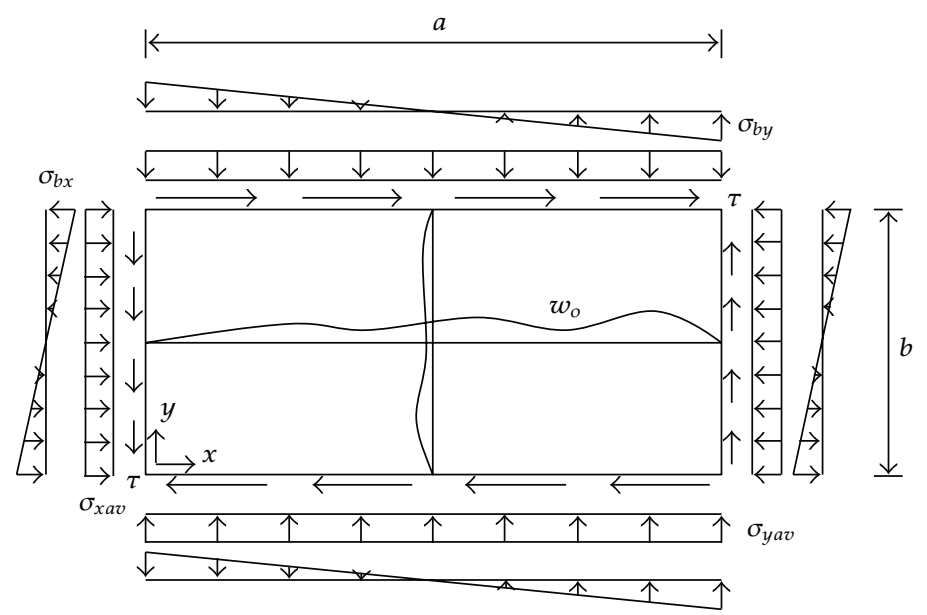

Figure 1: In-plane load components applied in a plate element.

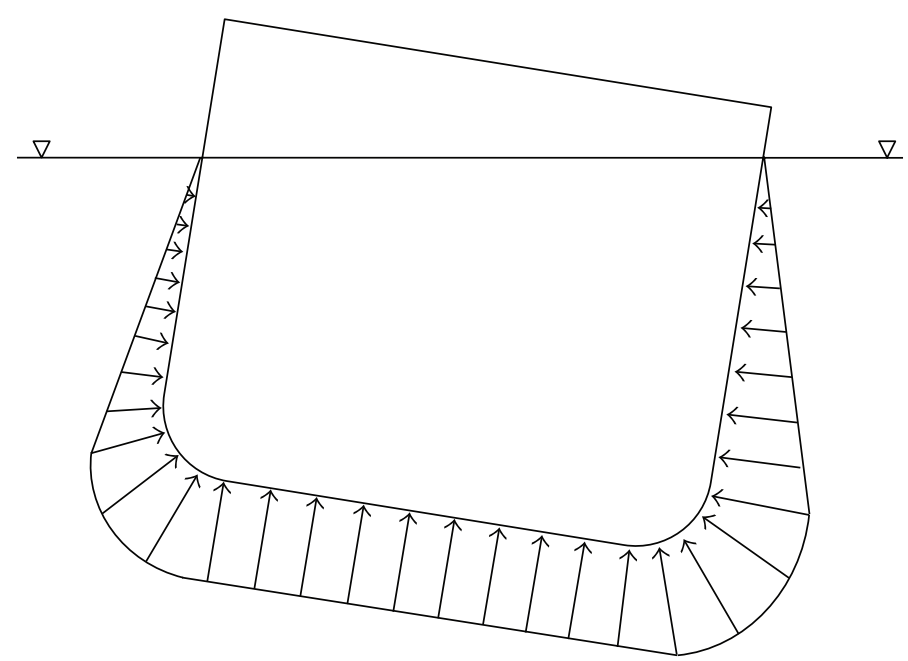

Figure 2: Roll motion of a vessel causing nonuniformity of lateral pressure load distribution in the hull cross-section.

of lateral pressure loads often becomes non-uniform as shown in Figure 3. For simplicity, however, the maritime industry usually applies a simplified design method in which the non-uniform distribution of lateral pressure loads is replaced by an equivalence of uniformly distributed lateral pressure loads with an average magnitude of applied pressure loads.

A large number of studies have been available in the literature, for example [1-6]. In the present paper, a mathematical algorithm is derived to analyze the elastic large deflection behavior, including buckling and postbuckling response, of plate elements under combined inplane and lateral pressure loads noted previously, with the emphasis on the non-uniformly distributed lateral pressure loads. The Galerkin method is applied to solve the nonlinear governing differential equations of elastic large deflection plate theory for plate elements. In the literature, the studies of metal plate buckling are also available, for example [7-13]. 


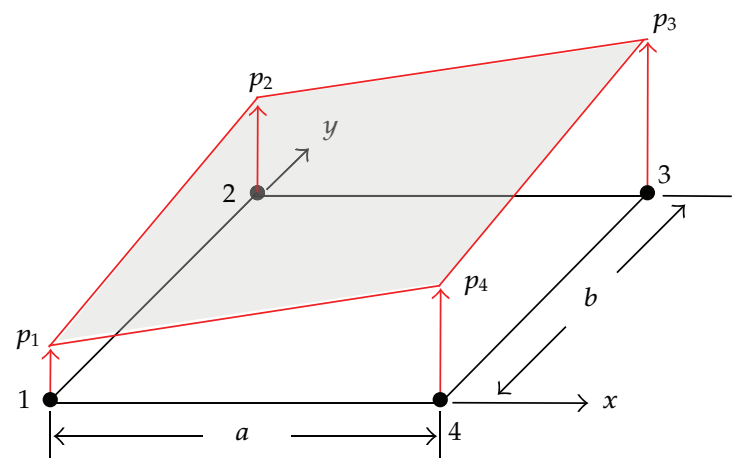

Figure 3: Non-uniformly distributed lateral pressure loads in a plate element.

\section{Elastic Large Deflection Analysis}

The elastic large deflection behavior of a plate element with initial imperfections can be analyzed by solving two differential equations, one representing the equilibrium condition and the other representing the compatibility condition [5]:

$$
\begin{aligned}
\Phi= & D\left(\frac{\partial^{4} w}{\partial x^{4}}+2 \frac{\partial^{4} w}{\partial x^{2} \partial y^{2}}+\frac{\partial^{4} w}{\partial y^{4}}\right) \\
& -t\left\{\frac{\partial^{2} F}{\partial y^{2}} \frac{\partial^{2}\left(w+w_{o}\right)}{\partial x^{2}}-2 \frac{\partial^{2} F}{\partial x \partial y} \frac{\partial^{2}\left(w+w_{o}\right)}{\partial x \partial y}+\frac{\partial^{2} F}{\partial x^{2}} \frac{\partial^{2}\left(w+w_{o}\right)}{\partial y^{2}}+\frac{p}{t}\right\}=0, \\
\frac{\partial^{4} F}{\partial x^{4}}+2 \frac{\partial^{4} F}{\partial x^{2} \partial y^{2}}+\frac{\partial^{4} F}{\partial y^{4}} & \\
& -E\left\{\left(\frac{\partial^{2} w}{\partial x \partial y}\right)^{2}-\frac{\partial^{2} w}{\partial x^{2}} \frac{\partial^{2} w}{\partial y^{2}}+2 \frac{\partial^{2} w_{o}}{\partial x \partial y} \frac{\partial^{2} w}{\partial x \partial y}-\frac{\partial^{2} w_{o}}{\partial x^{2}} \frac{\partial^{2} w}{\partial y^{2}}-\frac{\partial^{2} w}{\partial x^{2}} \frac{\partial^{2} w_{o}}{\partial y^{2}}\right\}=0 .
\end{aligned}
$$

Once the Airy stress function $F$ is defined, the membrane stress components at a certain position inside the plate may be expressed as follows:

$$
\begin{gathered}
\sigma_{x}=\frac{\partial^{2} F}{\partial y^{2}}-\frac{E z}{1-v^{2}}\left[\frac{\partial^{2} w}{\partial x^{2}}+v \frac{\partial^{2} w}{\partial y^{2}}\right], \\
\sigma_{y}=\frac{\partial^{2} F}{\partial x^{2}}-\frac{E z}{1-v^{2}}\left[\frac{\partial^{2} w}{\partial y^{2}}+v \frac{\partial^{2} w}{\partial x^{2}}\right], \\
\tau=\tau_{x y}=-\frac{\partial^{2} F}{\partial x \partial y}-\frac{E z}{2(1+v)} \frac{\partial^{2} w}{\partial x \partial y} .
\end{gathered}
$$


To apply the Galerkin method for solving the nonlinear governing differential equations, the added deflection $w$ and initial deflection $w_{o}$ are assumed as follows:

$$
\begin{gathered}
w=\sum_{m=1}^{M} \sum_{n=1}^{N} A_{m n} f_{m}(x) g_{n}(y), \\
w_{o}=\sum_{m=1}^{M} \sum_{n=1}^{N} A_{o m n} f_{m}(x) g_{n}(y),
\end{gathered}
$$

where $f_{m}(x)$ and $g_{n}(y)$ are functions which satisfy the boundary conditions for the plate. $A_{m n}$ and $A_{\text {omn }}$ are unknown and known deflection coefficients, respectively.

Equations (2.4) and (2.5) are substituted into (2.2) to obtain the stress function $F$. In this case, the particular solution $F_{P}$ of the stress function can be expressed as follows:

$$
F_{P}=\sum_{r=1}^{R} \sum_{s=1}^{S} K_{r s} p_{r}(x) q_{s}(y)
$$

where the coefficients $K_{r s}$ will be second-order functions with regard to the unknown deflection coefficients $A_{m n}$.

With the homogeneous solution $F_{H}$ of the stress function which satisfies the applied loading condition, the complete stress function $F$ may be given by

$$
F=F_{H}+\sum_{r=1}^{R} \sum_{s=1}^{S} K_{r s} p_{r}(x) q_{s}(y)
$$

To compute the unknown coefficients $A_{m n}$, the Galerkin method is applied to the equilibrium equation (2.1), resulting in the following equation:

$$
\iiint_{v} \Phi f_{r}(x) g_{s}(y) d v o l=0, \quad r=1,2,3, \ldots, s=1,2,3, \ldots
$$

Equations (2.4), (2.5), and (2.7) into (2.8), and performing the integration over the whole volume of the plate, a set of third-order simultaneous equations with regard to the unknown coefficients $A_{m n}$ will be obtained.

\section{Application to the Elastic Large Deflection Analysis of Simply Supported Plates}

In the present paper, the procedure described in [5] is applied. It is assumed that the plate element is simply supported along the four edges where support members such 
as longitudinal stiffeners and transverse frames are located. The simply supported edge conditions for the plates should satisfy the following conditions

$$
\begin{aligned}
& w=0, \quad \frac{\partial^{2} w}{\partial y^{2}}+v \frac{\partial^{2} w}{\partial x^{2}}=0 \quad \text { at } y=0, b \\
& w=0, \quad \frac{\partial^{2} w}{\partial x^{2}}+v \frac{\partial^{2} w}{\partial y^{2}}=0 \quad \text { at } x=0, a .
\end{aligned}
$$

To satisfy the boundary condition, the added deflection function $w$ and the initial deflection $w_{o}$ can be assumed in Fourier series:

$$
\begin{gathered}
w_{o}=\sum_{m=1}^{M} \sum_{n=1}^{N} A_{\text {omn }} \sin \frac{m \pi x}{a} \sin \frac{n \pi y}{b}, \\
w=\sum_{m=1}^{M} \sum_{n=1}^{N} A_{m n} \sin \frac{m \pi x}{a} \sin \frac{n \pi y}{b},
\end{gathered}
$$

where $A_{m n}$ and $A_{\text {omn }}$ are the unknown and the known coefficients, respectively. The condition of combined loads, namely biaxial loads, biaxial inplane bending and edge shear and lateral pressure loads are given as follows:

$$
\begin{gathered}
\int_{0}^{b} \frac{\partial^{2} F}{\partial y^{2}} t d y=P_{x} \quad \text { at } x=0, a, \\
\int_{0}^{b} \frac{\partial^{2} F}{\partial y^{2}} t\left(y-\frac{b}{2}\right) d y=M_{x} \quad \text { at } x=0, a, \\
\int_{0}^{a} \frac{\partial^{2} F}{\partial x^{2}} t d x=P_{y} \quad \text { at } y=0, b, \\
\int_{0}^{a} \frac{\partial^{2} F}{\partial x^{2}} t\left(x-\frac{a}{2}\right) d x=M_{y} \quad \text { at } y=0, b, \\
\frac{\partial^{2} F}{\partial x \partial y}=-\tau \text { at all boundaries. }
\end{gathered}
$$

For simplicity in expressing the various functions, the following abbreviations are involving sine or cosine terms:

$$
\sin \frac{m \pi x}{a}=s x(m), \quad \sin \frac{n \pi y}{b}=s y(n), \quad \cos \frac{m \pi x}{a}=c x(m), \quad \cos \frac{n \pi y}{b}=c y(n) .
$$


To find the stress function $F$ which should satisfy (2.2), (3.2) and (3.3) are substituted into (2.2) and the following equation yields:

$$
\begin{aligned}
& \frac{\partial^{4} F}{\partial x^{4}}+2 \frac{\partial^{4} F}{\partial x^{2} \partial y^{2}}+\frac{\partial^{4} F}{\partial y^{4}} \\
&=\frac{E \pi^{4}}{4 a^{2} b^{2}} \sum_{m=1}^{M} \sum_{n=1}^{N} \sum_{k=1}^{K} \sum_{l=1}^{L}\left[\left\{A_{m n} A_{k l} m l(n k-m l)-A_{o m n} A_{k l}(n k-m l)^{2}\right\} \times c x(m-k) c y(n-l)\right. \\
&+\left\{A_{m n} A_{k l} m l(n k+m l)+A_{o m n} A_{k l}(n k+m l)^{2}\right\} \times c x(m-k) c y(n+l) \\
&+\left\{A_{m n} A_{k l} m l(n k+m l)+A_{o m n} A_{k l}(n k+m l)^{2}\right\} \times c x(m+k) c y(n-l) \\
&\left.+\left\{A_{m n} A_{k l} m l(n k-m l)-A_{o m n} A_{k l}(n k-m l)^{2}\right\} \times c x(m+k) c y(n+l)\right] .
\end{aligned}
$$

A particular solution $F_{P}$ for the Airy stress function can be obtained as follows:

$$
\begin{aligned}
F_{P}=\sum_{m=1}^{M} \sum_{n=1}^{N} \sum_{k=1}^{K} \sum_{l=1}^{L}\{ & B_{1}(m, n, k, l) \times c x(m-k) c y(n-l) \\
& +B_{2}(m, n, k, l) \times c x(m-k) c y(n+l) \\
+ & B_{3}(m, n, k, l) \times c x(m+k) c y(n-l) \\
+ & \left.B_{4}(m, n, k, l) \times c x(m+k) c y(n+l)\right\} .
\end{aligned}
$$

Equation (3.7) is substituted into (2.2), the coefficients $B_{1}, B_{2}, B_{3}$ and $B_{4}$, as follows:

$$
\begin{aligned}
& B_{1}=\frac{E \alpha^{2}}{4} \times \frac{A_{m n} A_{k l} m l(n k-m l)-A_{o m n} A_{k l}(n k-m l)^{2}}{\left[(m-k)^{2}+\alpha^{2}(n-l)^{2}\right]^{2}}, \\
& B_{2}=\frac{E \alpha^{2}}{4} \times \frac{A_{m n} A_{k l} m l(n k+m l)+A_{o m n} A_{k l}(n k+m l)^{2}}{\left[(m-k)^{2}+\alpha^{2}(n+l)^{2}\right]^{2}}, \\
& B_{3}=\frac{E \alpha^{2}}{4} \times \frac{A_{m n} A_{k l} m l(n k+m l)+A_{o m n} A_{k l}(n k+m l)^{2}}{\left[(m+k)^{2}+\alpha^{2}(n-l)^{2}\right]^{2}}, \\
& B_{4}=\frac{E \alpha^{2}}{4} \times \frac{A_{m n} A_{k l} m l(n k-m l)-A_{o m n} A_{k l}(n k-m l)^{2}}{\left[(m+k)^{2}+\alpha^{2}(n+l)^{2}\right]^{2}} .
\end{aligned}
$$


Equation (3.8) is substituted into (3.7), and a particular solution $F_{P}$ is obtained as follows:

$$
\begin{aligned}
F_{P}=\frac{E \alpha^{2}}{4} \sum_{m=1}^{M} \sum_{n=1}^{N} \sum_{k=1}^{K} \sum_{l=1}^{L}\left\{\frac{A_{m n} A_{k l} m l(n k-m l)-A_{o m n} A_{k l}(n k-m l)^{2}}{\left[(m-k)^{2}+\alpha^{2}(n-l)^{2}\right]^{2}} \times c x(m-k) c y(n-l)\right. \\
+\frac{A_{m n} A_{k l} m l(n k+m l)+A_{o m n} A_{k l}(n k+m l)^{2}}{\left[(m-k)^{2}+\alpha^{2}(n+l)^{2}\right]^{2}} \times c x(m-k) c y(n+l) \\
+\frac{A_{m n} A_{k l} m l(n k+m l)+A_{o m n} A_{k l}(n k+m l)^{2}}{\left[(m+k)^{2}+\alpha^{2}(n-l)^{2}\right]^{2}} \times c x(m+k) c y(n-l) \\
\left.+\frac{A_{m n} A_{k l} m l(n k-m l)-A_{o m n} A_{k l}(n k-m l)^{2}}{\left[(m+k)^{2}+\alpha^{2}(n+l)^{2}\right]^{2}} \times c x(m+k) c y(n+l)\right\} .
\end{aligned}
$$

By considering the condition of load application, the homogeneous solution $F_{H}$ for the stress function is given by

$$
F_{H}=\left(\sigma_{x a v}+\sigma_{r x}\right) \frac{y^{2}}{2}+\left(\sigma_{y a v}+\sigma_{r y}\right) \frac{y^{2}}{2}-M_{x} \frac{y^{2}(2 y-3 b)}{b^{3} t}-M_{y} \frac{x^{2}(2 x-3 a)}{a^{3} t}-\tau_{x y} x y .
$$

The Airy stress function $F$ is then expressed by the sum of the particular and homogeneous solution as follows:

$$
F=F_{P}+F_{H}
$$

To compute the unknown coefficients $A_{m n}$, the Galerkin method is applied to (2.1) as follows:

$$
\iiint_{v} \Phi(x, y, z) s x(i) s y(j) d v o l=0, \quad i=1,2,3, \ldots, j=1,2,3, \ldots
$$


Substitution of (3.11) into (2.1) and then (2.1) to (3.12) after a derivation, a set of third order simultaneous equations for the unknown deflection coefficients $A_{m n}$ is obtained, as follows:

$$
\begin{aligned}
& \sum_{m=1}^{M} \sum_{n=1}^{N} \sum_{k=1}^{K} \sum_{l=1}^{L} \sum_{r=1}^{R} \sum_{s=1}^{S} A_{m n} A_{k l} A_{r s} \times \frac{E \alpha^{2} \pi^{4}(-t)}{4 a^{2} b^{2}}\left(R_{1}+R_{2}+R_{3}+R_{4}-2 R_{5}-2 R_{6}-2 R_{7}-2 R_{8}\right) \\
& +\sum_{m=1}^{M} \sum_{n=1}^{N} \sum_{k=1}^{K} \sum_{l=1}^{L} A_{m n} A_{k l} \times \frac{E \alpha^{2} \pi^{4}(-t)}{4 a^{2} b^{2}} \sum_{r=1}^{R} \sum_{s=1}^{S} A_{o r s}\left(R_{1}+R_{2}+R_{3}+R_{4}-2 R_{5}-2 R_{6}-2 R_{7}-2 R_{8}\right) \\
& +\sum_{k=1}^{K} \sum_{l=1}^{L} \sum_{r=1}^{R} \sum_{s=1}^{S} A_{k l} A_{r s} \times \frac{E \alpha^{2} \pi^{4}(-t)}{4 a^{2} b^{2}} \sum_{m=1}^{M} \sum_{n=1}^{N} A_{o m n}\left(R_{9}+R_{10}-R_{11}-R_{12}+2 R_{13}-2 R_{14}-2 R_{15}+2 R_{16}\right) \\
& +\sum_{k=1}^{K} \sum_{l=1}^{L} A_{k l} \times \frac{E \alpha^{2} \pi^{4}(-t)}{4 a^{2} b^{2}} \sum_{m=1}^{M} \sum_{n=1}^{N} \sum_{r=1}^{R} \sum_{s=1}^{S} A_{\text {omn }}\left(R_{9}+R_{10}-R_{11}-R_{12}+2 R_{13}-2 R_{14}-2 R_{15}+2 R_{16}\right) \\
& +\sum_{m=1}^{M} \sum_{n=1}^{N} A_{m n} \times D \pi^{4}\left(\frac{m^{2}}{a^{2}}+\frac{n^{2}}{b^{2}}\right)^{2} F_{1}(i, j, m, n) \\
& +\sum_{m=1}^{M} \sum_{n=1}^{N} A_{m n} \times(-t)\left\{\frac{m^{2} \pi^{2}}{a^{2}}\left[\left(\sigma_{x a v}+\sigma_{r x}-\frac{6}{b^{2} t} M_{x}\right) \times F_{1}(i, j, m, n)+\frac{12}{b^{3} t} M_{x} \times F_{2}(i, j, m, n)\right]\right. \\
& +\frac{n^{2} \pi^{2}}{b^{2}}\left[\left(\sigma_{y a v}+\sigma_{r y}-\frac{6}{a^{2} t} M_{y}\right) \times F_{1}(i, j, m, n)+\frac{12}{a^{3} t} M_{y} \times F_{3}(i, j, m, n)\right] \\
& \left.+\frac{2 \tau \pi^{4} m n}{a b} \times F_{4}(i, j, m, n)\right\} \\
& +\sum_{m=1}^{M} \sum_{n=1}^{N} A_{o m n} \times(-t)\left\{\frac{m^{2} \pi^{2}}{a^{2}}\left[\left(\sigma_{x a v}+\sigma_{r x}-\frac{6}{b^{2} t} M_{x}\right) \times F_{1}(i, j, m, n)+\frac{12}{b^{3} t} M_{x} \times F_{2}(i, j, m, n)\right]\right. \\
& +\frac{n^{2} \pi^{2}}{b^{2}}\left[\left(\sigma_{y a v}+\sigma_{r y}-\frac{6}{a^{2} t} M_{y}\right) \times F_{1}(i, j, m, n)+\frac{12}{a^{3} t} M_{y} \times F_{3}(i, j, m, n)\right] \\
& \left.+\frac{2 \tau \pi^{4} m n}{a b} \times F_{4}(i, j, m, n)\right\} \\
& -F_{5}(i, j) \times q=0,
\end{aligned}
$$

where $q=$ lateral pressure loads, and the coefficients $R_{1}$ to $R_{16}$ and $F_{1}$ to $F_{5}$ are given in Appendix.

In (3.13), lateral pressure $q$ will take the following form in general.

$$
q=\frac{p_{4}-p_{1}}{a} x+\frac{p_{2}-p_{1}}{b} y+\frac{p_{1}-p_{2}+p_{3}-p_{4}}{a b} x y+p_{1} .
$$

When uniform pressure loads are applied, that is, with $p_{1}=p_{2}=p_{3}=p_{4}, q=p_{1}$ is taken as constant value. 

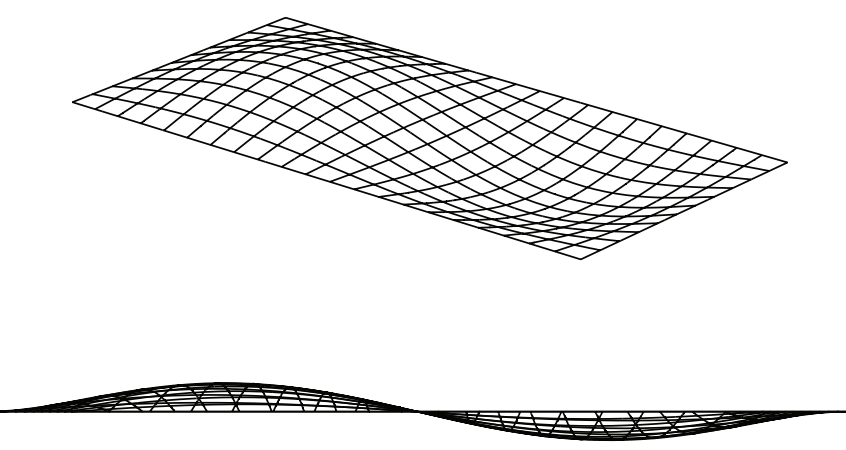

Figure 4: Configuration of the initial deflection in the plate.

\section{Applied Examples and Discussion}

Some applied examples are now presented. A simply supported rectangular plate of $a \times b \times t=$ $1660 \mathrm{~mm} \times 830 \times 16 \mathrm{~mm}$ under uniaxial compression and lateral pressure loads is considered, in which lateral pressure loads are applied first, followed by the monotonical application of axial compressive loads. The elastic modulus and Poisson's ratio are $E=205.8 \mathrm{GPa}$ and $v=0.3$. The elastic buckling strength $\sigma_{x E}$ of this plate under uniaxial compression in the $x$ direction is determined as follows:

$$
\sigma_{x E}=k \frac{\pi^{2} E}{12\left(1-v^{2}\right)}\left(\frac{t}{b}\right)^{2}
$$

where $k=$ buckling coefficient which is taken as $k=4$.

Also, the lateral pressure loads are taken as $p_{1}=p_{2}=p_{a}$ and $p_{3}=p_{4}=p_{b}$ in the present examples. The initial deflection of the plate is selected as follows (see Figure 4 ):

$$
w_{0}=A_{021} \sin \frac{2 \pi x}{a} \sin \frac{\pi y}{b}
$$

where $A_{021}=0.05 t$.

The added deflection of the plate due to applied loads can be expressed as follows.

$$
w=\sum_{m=1}^{M} \sum_{n=1}^{N} A_{m n} \sin \frac{m \pi x}{a} \sin \frac{n \pi y}{b} .
$$

In the present study, the maximum number of amplitudes in the shorter direction of the plate is assumed as $N=1$, but that in the longer direction of the plate is varied to investigate the best selection of $M$ in terms of the accuracy and efficiency of the computations.

Figures $5(\mathrm{a})$ to $5(\mathrm{~d})$ present the relation between the axial compressive loads versus total deflection at the center of the plate, that is, at $x=a / 2$ and $y=b / 2$, where the magnitude of lateral pressure loads and the maximum number of deflection amplitudes are varied. It is found that $M=5$ may be sufficient enough in terms of accuracy and efficiency of the resulting computations. Table 1 represents the deformed shapes of the plate with different 


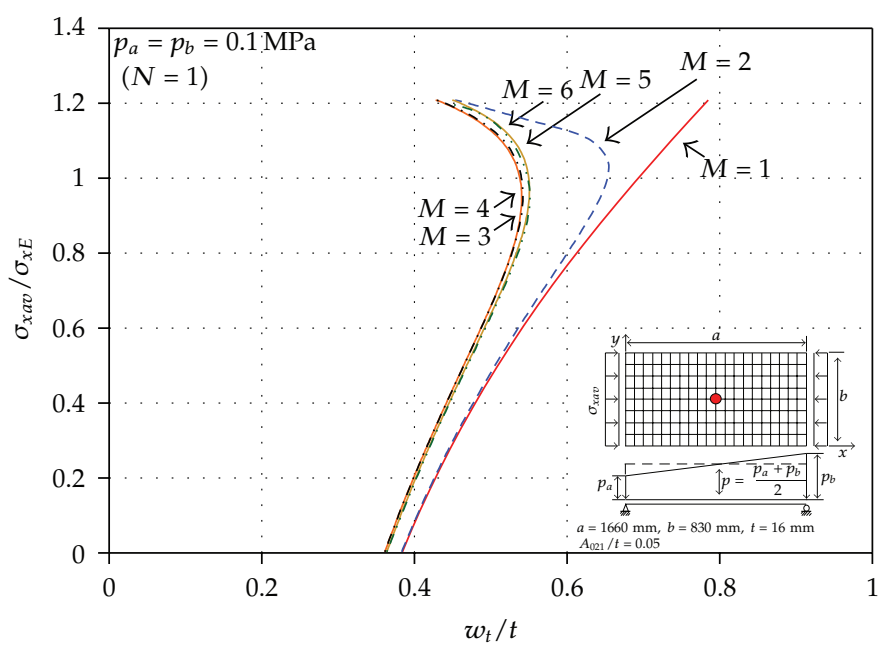

(a)

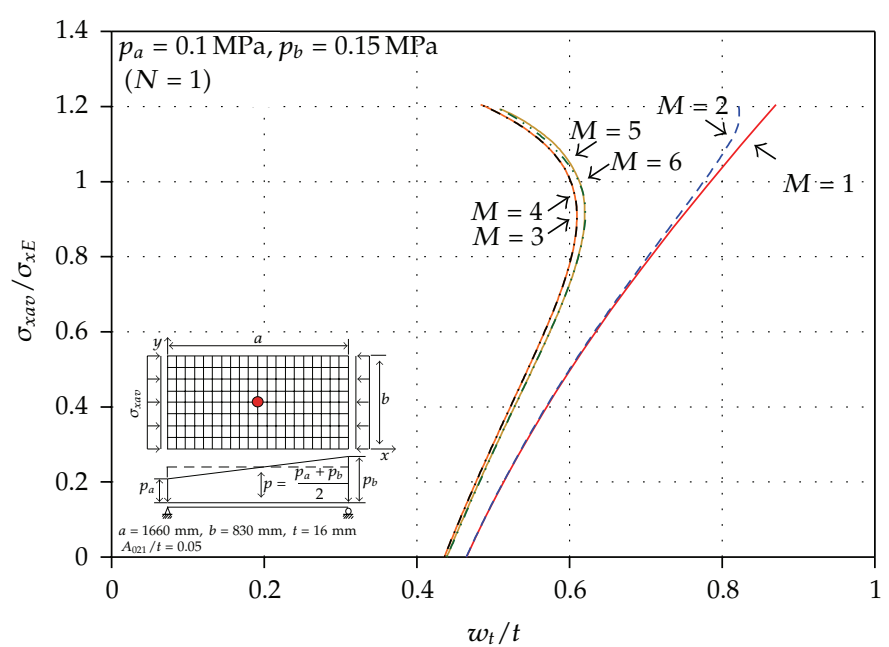

(b)

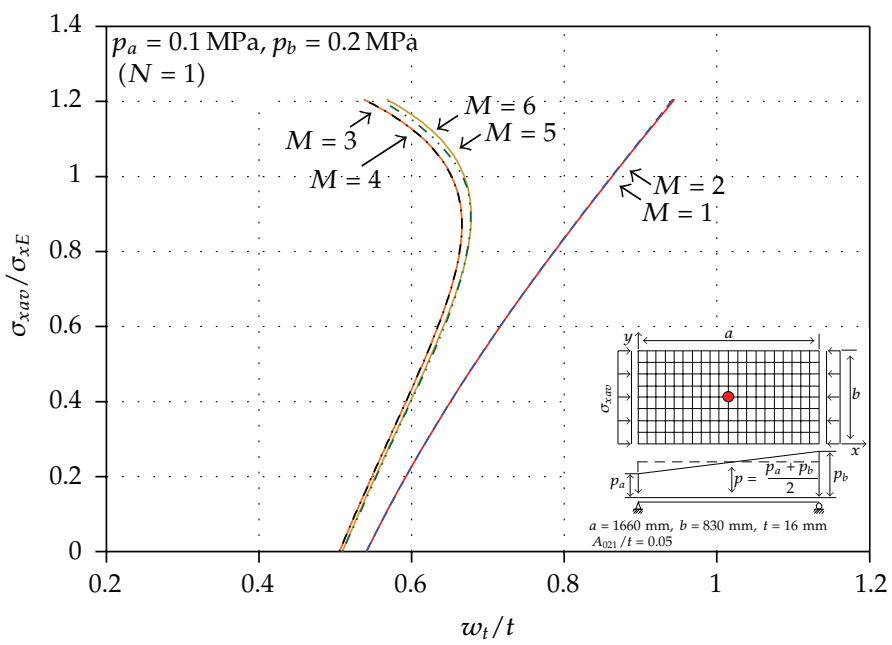

(c)

Figure 5: Continued. 


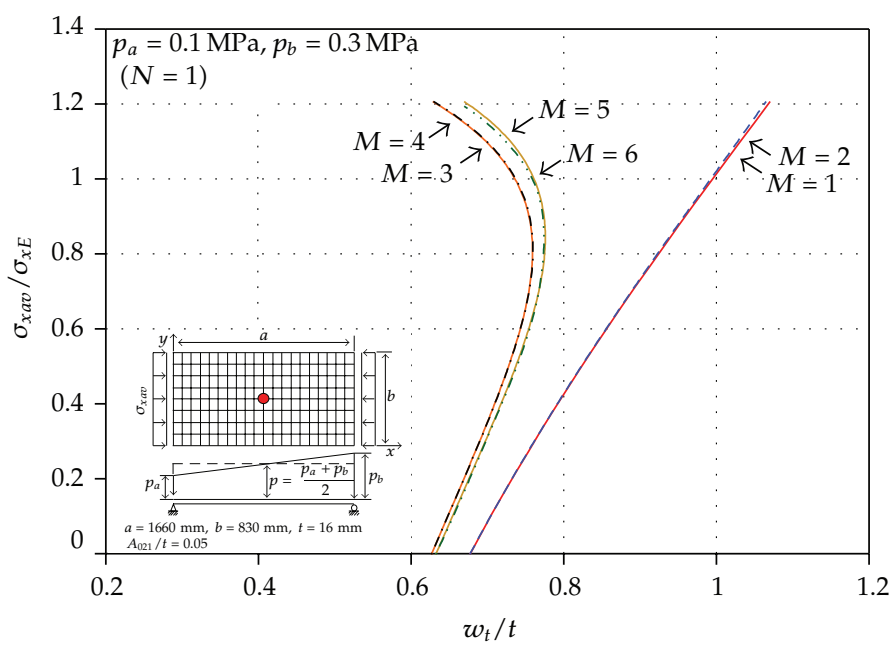

(d)

Figure 5: (a) The axial compressive load versus total deflection curves at $p_{a}=p_{b}=0.1 \mathrm{MPa}$. (b) The axial compressive load versus total deflection curves at $p_{a}=0.1 \mathrm{MPa}$ and $p_{b}=0.15 \mathrm{MPa}$. (c) The axial compressive load versus total deflection curves at $p_{a}=0.1 \mathrm{MPa}$ and $p_{b}=0.2 \mathrm{MPa}$. (d) The axial compressive load versus total deflection curves at $p_{a}=0.1 \mathrm{MPa}$ and $p_{b}=0.3 \mathrm{MPa}$.

magnitudes or shapes of lateral pressure loads when $\sigma_{x a v} / \sigma_{x E}=0$ or 1.2 where $M=5$ is taken. It is observed that the deflection modes of the plate are changed due to the application of axial compressive loads. This is because the plate tends to deflect as per the original buckling pattern associated with axial compressive loads.

Figure 6 presents a comparison of the elastic large deflection responses of the plate between non-uniform and uniform pressure loads, the latter being taken as the average value of the pressure loads as per the current practice of the maritime industry.

It is found that the current practice of the maritime industry with an average magnitude of applied pressure loads underestimates the lateral deflection of the plate compared to the real condition of the pressure load application, that is, with a non-uniform distribution. The difference between the two cases becomes larger and larger as the nonuniformity of lateral pressure loads becomes more significant.

The underestimation of the plate deflection due to external pressure loads gives rise to the overestimation of plate strength performance which may lead to unsafe design at optimistic side.

\section{Concluding Remarks}

The aim of the present paper has been to analyze the elastic large deflection behavior of metal plates subject to combined inplane and lateral pressure loads. When lateral pressure loads applied in plate elements are non-uniform, the current practice of the maritime industry applies some simplified design methods in which the non-uniform pressure distribution in the plates is replaced by an equivalence of uniform pressure distribution.

In the present paper, the Galerkin method was used to solve the nonlinear governing differential equations of plate elements under non-uniformly distributed lateral pressure loads in addition to inplane loads. Some applied examples are presented, demonstrating that 
Table 1: Deformed shapes of the plate when $M=5$.

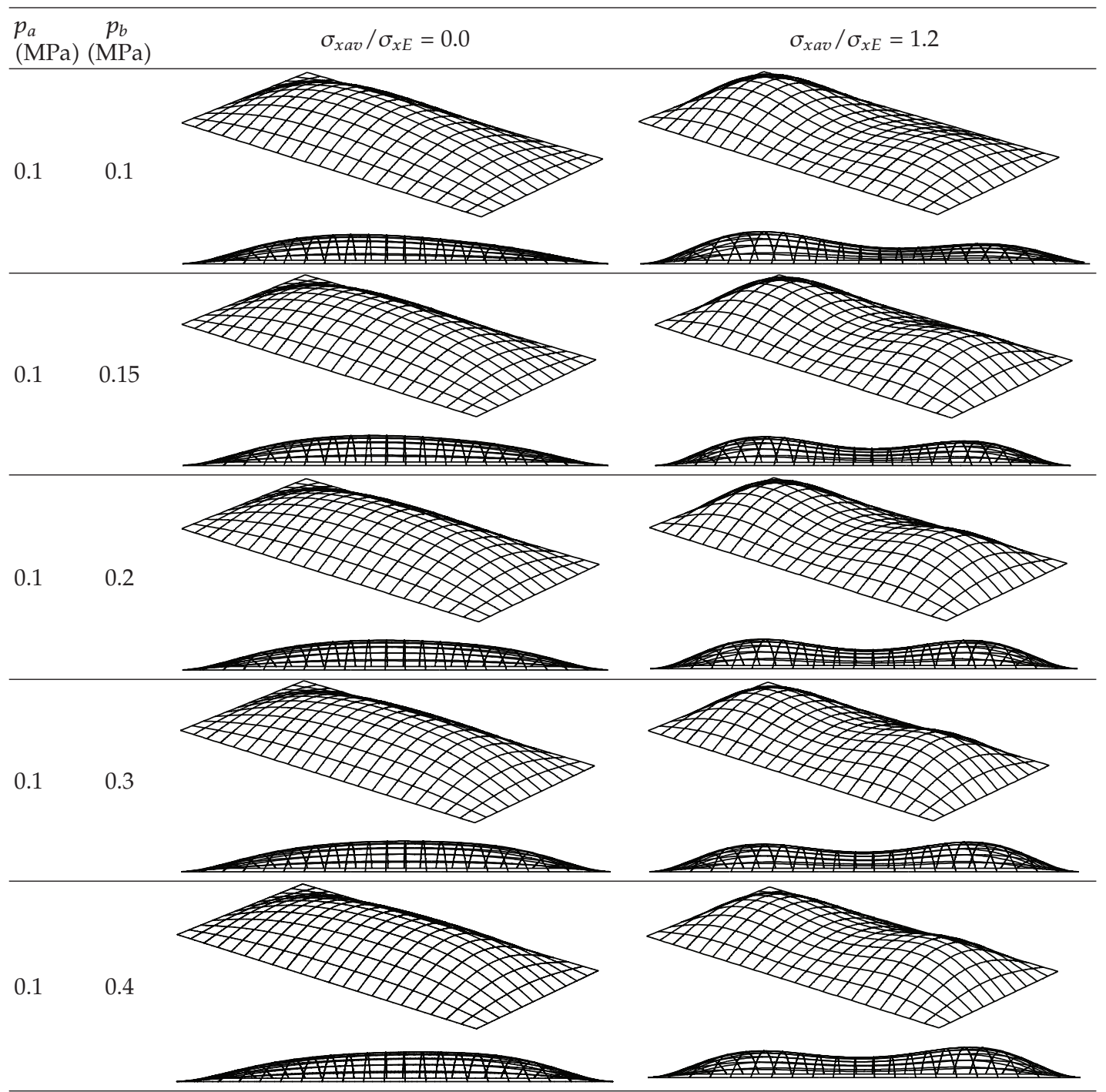

the current practice of the maritime industry, that is, with an average magnitude of applied pressure loads as an equivalence, results in a great underestimation of lateral deflection calculations when the non-uniformity of lateral pressure loads becomes more significant. The underestimation of the plate deflection may lead to unsafe design of plates at optimistic side.

Thin plates buckle in elastic regime, while stocky plates may buckle in elastic-plastic or plastic regime. The present paper deals with elastic behavior only, and further studies are then recommended to take into account the effect of plasticity which is dominant in thick plates. Also, it is highly desirable for practical design purpose to develop a simpler method or design formulation. 
Journal of Applied Mathematics

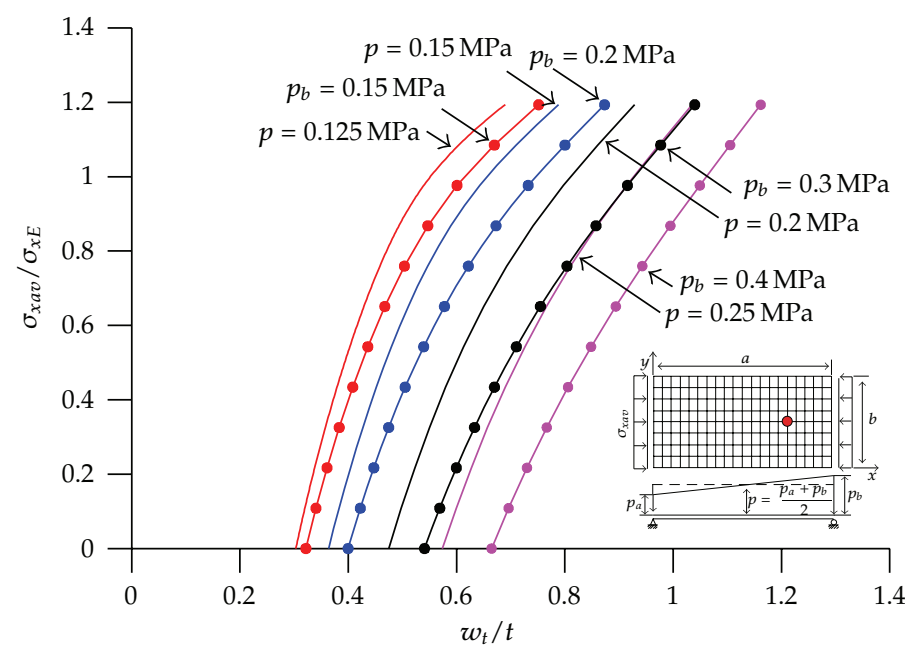

$M=5, N=1$

$a=1660 \mathrm{~mm}, b=830 \mathrm{~mm}, t=16 \mathrm{~mm}$ $A_{021} / t=0.05$

$p_{a}=0.1 \mathrm{MPa}$

Line: uniform lateral pressure

Line with dot: nonuniform lateral pressure

Figure 6: The axial compressive loads versus total deflection at $x=3 a / 4$ and $y=b / 2$.

\section{Appendix}

The coefficients $R_{1}$ to $R_{16}$ and $F_{1}$ to $F_{5}$ are given as follows:

$$
\begin{gathered}
F_{1}(i, j, m, n)=\int_{0}^{t} \int_{0}^{b} \int_{0}^{a} s x(m) s y(n) s x(i) s y(j) d x d y d z, \\
F_{2}(i, j, m, n)=\int_{0}^{t} \int_{0}^{b} \int_{0}^{a} y \cdot s x(m) s y(n) s x(i) s y(j) d x d y d z, \\
F_{3}(i, j, m, n)=\int_{0}^{t} \int_{0}^{b} \int_{0}^{a} x \cdot s x(m) s y(n) s x(i) s y(j) d x d y d z, \\
F_{4}(i, j, m, n)=\int_{0}^{t} \int_{0}^{b} \int_{0}^{a} c x(m) c y(n) s x(i) s y(j) d x d y d z, \\
F_{5}(i, j)=\int_{0}^{t} \int_{0}^{b} \int_{0}^{a} s x(i) s y(j) d x d y d z, \\
\quad(m+k)^{2} s^{2}+(n+l)^{2} r^{2} \\
{\left[(m+k)^{2}+\alpha^{2}(n+l)^{2}\right]} \\
\left.\times \int_{0}^{t} \int_{0}^{b} \int_{0}^{a} c x(m+k) c y l\right)
\end{gathered}
$$




$$
\begin{aligned}
& R_{2}(i, j, m, n, k, l, r, s)=\frac{(m+k)^{2} s^{2}+(n-l)^{2} r^{2}}{\left[(m+k)^{2}+\alpha^{2}(n-l)^{2}\right]} m l(n k+m l) \\
& \times \int_{0}^{t} \int_{0}^{b} \int_{0}^{a} c x(m+k) c y(n-l) s x(r) s y(s) s x(i) s y(j) d x d y d z, \\
& R_{3}(i, j, m, n, k, l, r, s)=\frac{(m-k)^{2} s^{2}+(n+l)^{2} r^{2}}{\left[(m-k)^{2}+\alpha^{2}(n+l)^{2}\right]} m l(n k+m l) \\
& \times \int_{0}^{t} \int_{0}^{b} \int_{0}^{a} c x(m-k) c y(n+l) s x(r) s y(s) s x(i) s y(j) d x d y d z, \\
& R_{4}(i, j, m, n, k, l, r, s)=\frac{(m-k)^{2} s^{2}+(n-l)^{2} r^{2}}{\left[(m-k)^{2}+\alpha^{2}(n-l)^{2}\right]} m l(n k-m l) \\
& \times \int_{0}^{t} \int_{0}^{b} \int_{0}^{a} c x(m-k) c y(n-l) s x(r) s y(s) s x(i) s y(j) d x d y d z, \\
& R_{5}(i, j, m, n, k, l, r, s)=\frac{(m+k)(n+l)}{\left[(m+k)^{2}+\alpha^{2}(n+l)^{2}\right]^{2}} m l r s(n k-m l) \\
& \times \int_{0}^{t} \int_{0}^{b} \int_{0}^{a} s x(m+k) s y(n+l) c x(r) c y(s) s x(i) s y(j) d x d y d z, \\
& R_{6}(i, j, m, n, k, l, r, s)=\frac{(m+k)(n-l)}{\left[(m+k)^{2}+\alpha^{2}(n-l)^{2}\right]^{2}} m l r s(n k+m l) \\
& \times \int_{0}^{t} \int_{0}^{b} \int_{0}^{a} s x(m+k) s y(n-l) c x(r) c y(s) s x(i) s y(j) d x d y d z, \\
& R_{7}(i, j, m, n, k, l, r, s)=\frac{(m-k)(n+l)}{\left[(m-k)^{2}+\alpha^{2}(n+l)^{2}\right]^{2}} \operatorname{mlrs}(n k+m l) \\
& \times \int_{0}^{t} \int_{0}^{b} \int_{0}^{a} s x(m-k) s y(n+l) c x(r) c y(s) s x(i) s y(j) d x d y d z, \\
& R_{8}(i, j, m, n, k, l, r, s)=\frac{(m-k)(n-l)}{\left[(m-k)^{2}+\alpha^{2}(n-l)^{2}\right]^{2}} m l r s(n k-m l) \\
& \times \int_{0}^{t} \int_{0}^{b} \int_{0}^{a} s x(m-k) s y(n-l) c x(r) c y(s) s x(i) s y(j) d x d y d z, \\
& R_{9}(i, j, m, n, k, l, r, s)=\frac{(m+k)^{2} s^{2}+(n+l)^{2} r^{2}}{\left[(m+k)^{2}+\alpha^{2}(n+l)^{2}\right]}(n k-m l)^{2} \\
& \times \int_{0}^{t} \int_{0}^{b} \int_{0}^{a} c x(m+k) c y(n+l) s x(r) s y(s) s x(i) s y(j) d x d y d z,
\end{aligned}
$$


Journal of Applied Mathematics

$$
\begin{aligned}
& R_{10}(i, j, m, n, k, l, r, s)=\frac{(m+k)^{2} s^{2}+(n-l)^{2} r^{2}}{\left[(m+k)^{2}+\alpha^{2}(n-l)^{2}\right]}(n k+m l)^{2} \\
& \times \int_{0}^{t} \int_{0}^{b} \int_{0}^{a} c x(m+k) c y(n-l) s x(r) s y(s) s x(i) s y(j) d x d y d z, \\
& R_{11}(i, j, m, n, k, l, r, s)=\frac{(m-k)^{2} s^{2}+(n+l)^{2} r^{2}}{\left[(m-k)^{2}+\alpha^{2}(n+l)^{2}\right]}(n k+m l)^{2} \\
& \times \int_{0}^{t} \int_{0}^{b} \int_{0}^{a} c x(m-k) c y(n+l) s x(r) s y(s) s x(i) s y(j) d x d y d z, \\
& R_{12}(i, j, m, n, k, l, r, s)=\frac{(m-k)^{2} s^{2}+(n-l)^{2} r^{2}}{\left[(m-k)^{2}+\alpha^{2}(n-l)^{2}\right]}(n k-m l)^{2} \\
& \times \int_{0}^{t} \int_{0}^{b} \int_{0}^{a} c x(m-k) c y(n-l) s x(r) s y(s) s x(i) s y(j) d x d y d z, \\
& R_{13}(i, j, m, n, k, l, r, s)=\frac{(m+k)(n+l)}{\left[(m+k)^{2}+\alpha^{2}(n+l)^{2}\right]^{2}} r s(n k-m l)^{2} \\
& \times \int_{0}^{t} \int_{0}^{b} \int_{0}^{a} s x(m+k) s y(n+l) c x(r) c y(s) s x(i) s y(j) d x d y d z, \\
& R_{14}(i, j, m, n, k, l, r, s)=\frac{(m+k)(n-l)}{\left[(m+k)^{2}+\alpha^{2}(n-l)^{2}\right]^{2}} r s(n k+m l)^{2} \\
& \times \int_{0}^{t} \int_{0}^{b} \int_{0}^{a} s x(m+k) s y(n-l) c x(r) c y(s) s x(i) s y(j) d x d y d z, \\
& R_{15}(i, j, m, n, k, l, r, s)=\frac{(m-k)(n+l)}{\left[(m-k)^{2}+\alpha^{2}(n+l)^{2}\right]^{2}} r s(n k+m l)^{2} \\
& \times \int_{0}^{t} \int_{0}^{b} \int_{0}^{a} s x(m-k) s y(n+l) c x(r) c y(s) s x(i) s y(j) d x d y d z, \\
& R_{16}(i, j, m, n, k, l, r, s)=\frac{(m-k)(n-l)}{\left[(m-k)^{2}+\alpha^{2}(n-l)^{2}\right]^{2}} r s(n k-m l)^{2} \\
& \times \int_{0}^{t} \int_{0}^{b} \int_{0}^{a} s x(m-k) s y(n-l) c x(r) c y(s) s x(i) s y(j) d x d y d z .
\end{aligned}
$$

\section{Nomenclature}

$a$ : Length of the plate

$b$ : Breadth of the plate 


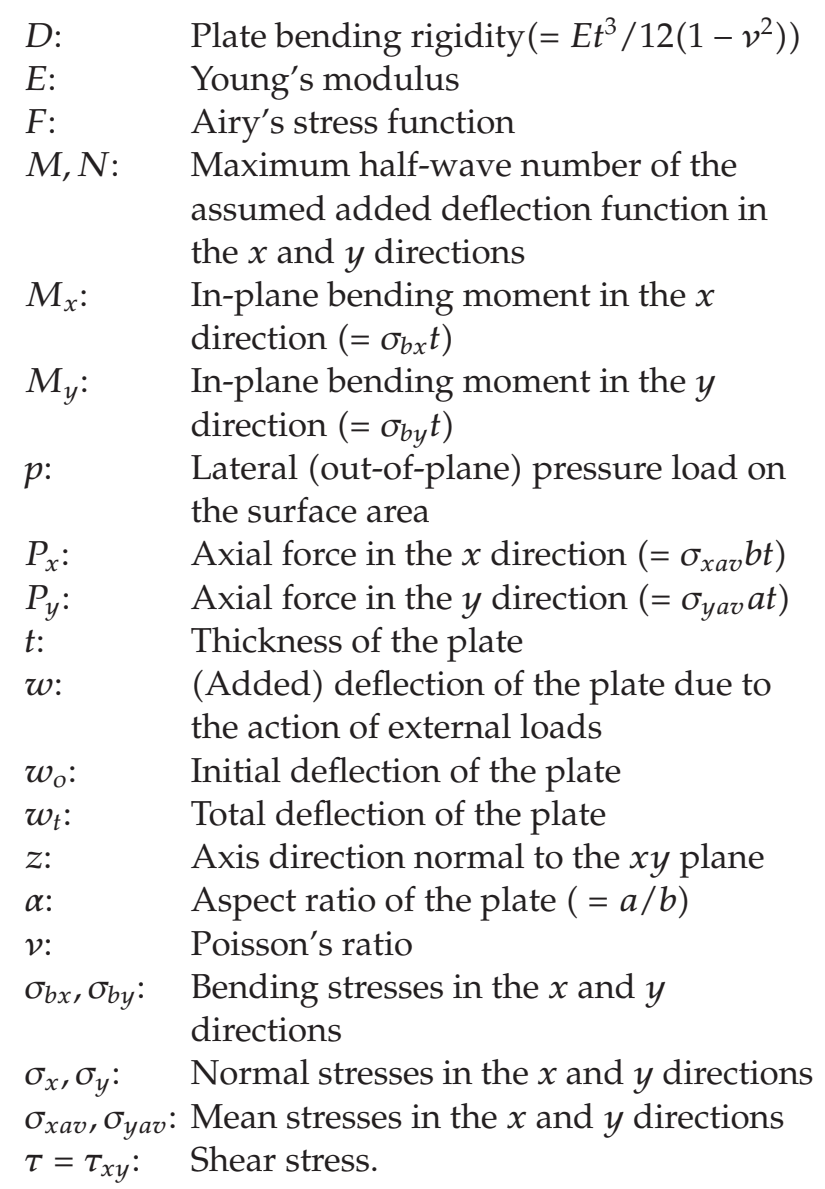

\section{Acknowledgments}

This research was supported by Basic Science Research Program through the National Research Foundation of Korea (NRF) funded by the Ministry of Education, Science and Technology (Grant no. K20903002030-11E0100-04610).

\section{References}

[1] R. Szilard, Theory and Analysis of Plates: Classical and Numerical Methods, Prentice Hall, Upper Saddle River, NJ, USA, 1974.

[2] S. P. Timoshenko and S. Woinowsky-Krieger, Theory of Plates and Shells, McGraw-Hill, London, UK, 1981.

[3] J. K. Paik and A. K. Thayamballi, Ultimate Limit State Design of Steel-Plated Structures, John Wiley \& Sons, Chichester, UK, 2003.

[4] O. F. Hughes and J. K. Paik, Ship Structural Analysis and Design, The Society of Naval Architects and Marine Engineers, New Jersey, NJ, USA, 2010.

[5] J. K. Paik, A. K. Thayamballi, S. K. Lee, and S. J. Kang, "Semi-analytical method for the elastic-plastic large deflection analysis of welded steel or aluminum plating under combined in-plane and lateral pressure loads," Thin-Walled Structures, vol. 39, no. 2, pp. 125-152, 2001.

[6] J. K. Paik and M. S. Lee, "A semi-analytical method for the elastic-plastic large deflection analysis of stiffened panels under combined biaxial compression/tension, biaxial in-plane bending, edge shear, and lateral pressure loads," Thin-Walled Structures, vol. 43, no. 3, pp. 375-410, 2005. 
[7] G. Wang, H. Sun, H. Peng, and R. Uemori, "Buckling and ultimate strength of plates with openings," Ships and Offshore Structures, vol. 4, no. 1, pp. 43-53, 2009.

[8] U. N. Kim, I. H. Choe, and J. K. Paik, "Buckling and ultimate strength of perforated plate panels subject to axial compression: experimental and numerical investigations with design formulations," Ships and Offshore Structures, vol. 4, no. 4, pp. 337-361, 2009.

[9] P. P. Chaithanya, P. K. Das, A. Crow, and S. Hunt, "The effect of distortion on the buckling strength of stiffened panels," Ships and Offshore Structures, vol. 5, no. 2, pp. 141-153, 2010.

[10] K. M. El-Sawy and M. I. Martini, "Stability of biaxially loaded square plates with single central holes," Ships and Offshore Structures, vol. 5, no. 4, pp. 283-293, 2010.

[11] S. Benson, J. Downes, and R. S. Dow, "Ultimate strength characteristics of aluminium plates for highspeed vessels," Ships and Offshore Structures, vol. 6, no. 1-2, pp. 67-80, 2011.

[12] J. K. Paik, "Ultimate strength of steel plates with a single circular hole under axial compressive loading along short edges," Ships and Offshore Structures, vol. 2, no. 4, pp. 355-360, 2007.

[13] M. S. Kumar, P. Alagusundaramoorthy, and R. Sundaravadivelu, "Interaction curves for stiffened panel with circular opening under axial and lateral loads," Ships and Offshore Structures, vol. 4, no. 2, pp. 133-143, 2009. 


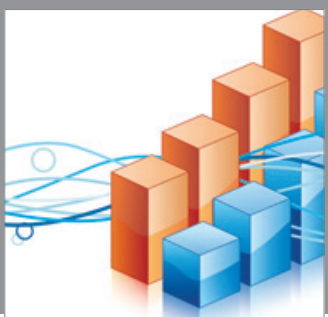

Advances in

Operations Research

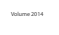

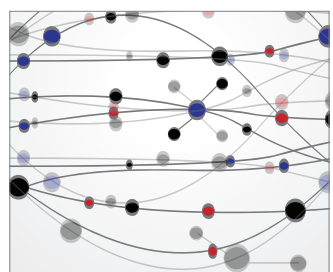

\section{The Scientific} World Journal
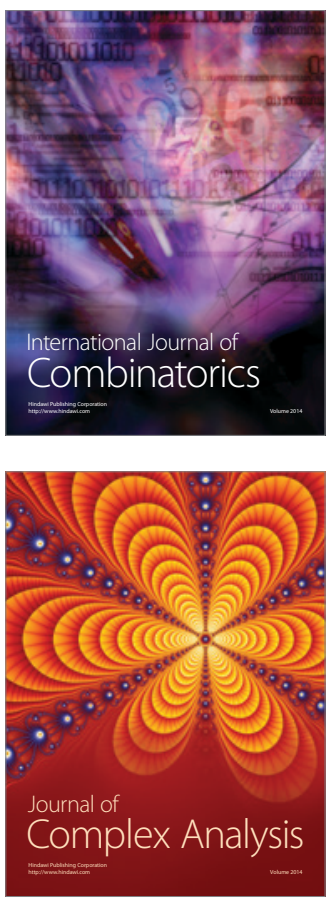

International Journal of

Mathematics and

Mathematical

Sciences
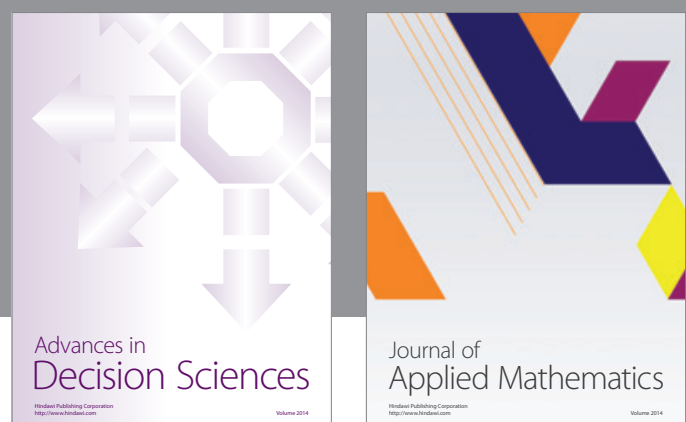

Journal of

Applied Mathematics
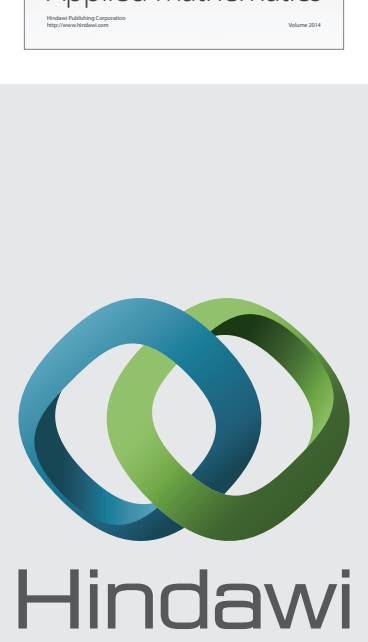

Submit your manuscripts at http://www.hindawi.com
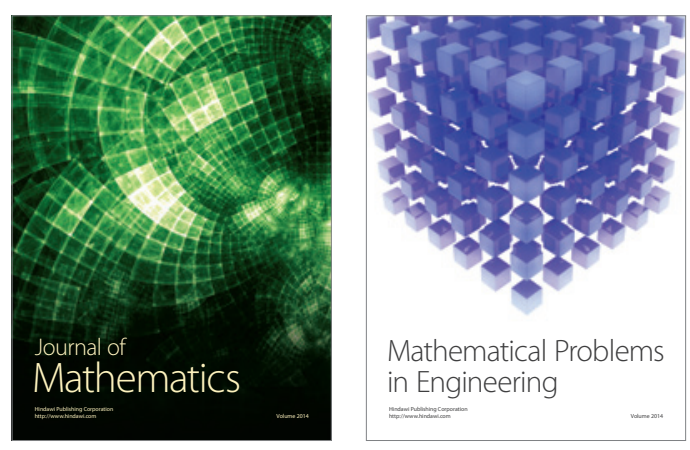

Mathematical Problems in Engineering
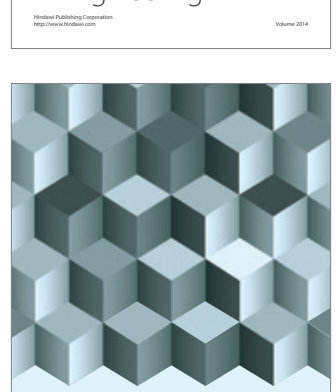

Journal of

Function Spaces
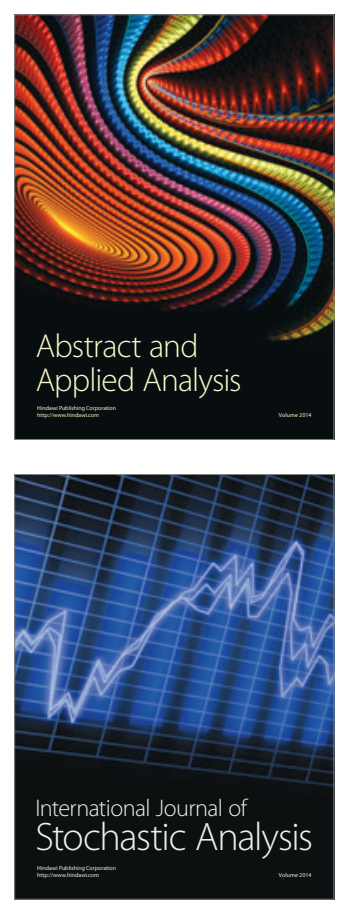

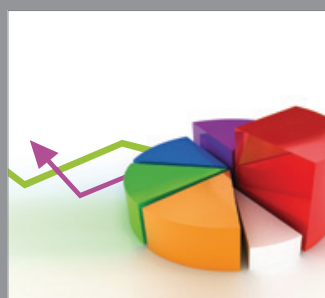

ournal of

Probability and Statistics

Promensencen
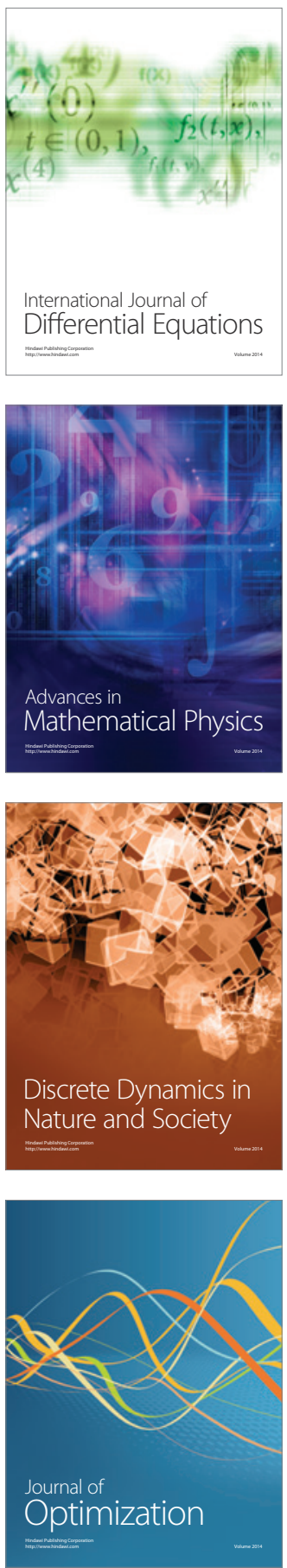